\title{
Circumcentered reflections method for wavelet feasibility problems
}
N. D. Dizon ${ }^{1}$
J. A. $\operatorname{Hogan}^{2}$
S. B. Lindstrom ${ }^{3}$

(Received 12 2020; revised 14 August 2021)

\begin{abstract}
We introduce a two-stage global-then-local search method for solving feasibility problems. The approach pairs the advantageous global tendency of the Douglas-Rachford method to find a basin of attraction for a fixed point, together with the local tendency of the circumcentered reflections method to perform faster within such a basin. We experimentally demonstrate the success of the method for solving nonconvex problems in the context of wavelet construction formulated as a feasibility problem.
\end{abstract}

\section{Contents}

\section{Introduction}

DOI:10.21914/anziamj.v62.16118, (C) Austral. Mathematical Soc. 2022. Published 2022-01-09, as part of the Proceedings of the 19th Biennial Computational Techniques and Applications Conference. ISSN 1445-8810. (Print two pages per sheet of paper.) Copies of this article must not be made otherwise available on the internet; instead link directly to the DOI for this article. 
2 Wavelet construction problem

3 DR and CRM for wavelet construction

C103

4 The two-stage DR-CRM search algorithm

C107

5 Conclusion

C108

\section{Introduction}

Wavelet construction has been recently formulated as a feasibility problem [9, $10,11]$. In harmonic analysis, wavelets are signal processing tools that define a transform which overcomes the shortcomings of the classical Fourier transform in time-frequency analysis. In optimisation, to solve a feasibility problem is to find a point on the intersection of a finite family of sets. By formulating wavelet architecture as a feasibility problem, the construction of a wavelet translates to the task of finding a point on the intersection of closed constraint sets arising from the conditions and properties that the wavelet must satisfy.

Wavelets are functions whose shifts and dilates form an orthonormal basis for the ambient function space. The multiresolution structure and compact support properties are crucial ingredients in the development of fast transform techniques [6]. Additionally, the regularity of a wavelet dictates the sparsity of coefficients in the decomposition. The earliest examples of smooth compactly supported wavelets on the line were achieved by Daubechies [6] through the multiresolution analysis (MRA) introduced by Mallat [15] and Meyer [16]. However, the techniques employed by Daubechies (especially those based on complex analysis) do not apply to higher dimensions. With the recent introduction of the feasibility approach by Franklin, Hogan, and Tam, nontensorial constructions in higher dimensions become much more accessible [9, 10, 11]. Moreover, the feasibility approach readily accounts for other design criteria, including symmetry and cardinality [7].

The method of alternating projections and the Douglas-Rachford (DR) method 
are two well-known projection algorithms that solve two-set feasibility problems. The DR method is notable for its dexterity in solving convex and nonconvex feasibility problems, including Sudoku puzzles, matrix completion, and phase retrieval $[1,13]$. When applied to nonconvex feasibility problems involving hypersurfaces, DR iterates often exhibit an initial search for a basin of attraction to a fixed point, followed thereafter by a spiraling pattern within the basin $[5,4,12,14]$; this spiraling is associated with slower convergence. With the goal of obviating excessive spiralling by taking a clever step towards the feasible point, Behling, Bello Cruz, and Santos [2] introduced the idea of circumcentering the substeps of the DR method for intersecting affine subspaces. This has since come to be known as the circumcentered reflections method (CRM) [3]. The method involves the computation of a circumcenter (of successive reflections) which may not be defined outside the context of intersecting affine subspaces. For the broader context of nonconvex feasibility problems, Dizon, Hogan, and Lindstrom [8] introduced a piecewise modification that computes a step of CRM when the circumcenter construction is well-defined, and reverts to a normal DR step when it is not. This construction is always well-defined.

In this article, we introduce a heuristic for solving feasibility problems by forming a two-stage DR-CRM, where we take CRM to mean the always-welldefined version. We illustrate that assembling DR and CRM together combines the robustness of DR in global searches and the speed of CRM during a local search. We compare performance of this two-stage method with a full DR approach in the context of wavelet feasibility problems.

The rest of the article is organised as follows. Section 2 recalls the wavelet feasibility problem. Section 3 revisits DR and CRM, thereafter comparing their performance in solving wavelet feasibility problems. In Section 4, we assemble the two-stage DR-CRM approach, and assess its performance in dealing with the feasibility problem at hand. 


\section{Wavelet construction problem}

The construction of wavelet orthonormal bases is traditionally achieved by finding a scaling function-wavelet pair $(\varphi, \psi)$ that satisfies conditions arising from the MRA. For wavelets on the line, the construction reduces to finding a matrix-valued function $\mathrm{U}(\xi): \mathbb{R} \rightarrow \mathbb{C}^{2 \times 2}$ of the form

$$
\mathrm{u}(\xi)=\left[\begin{array}{cc}
\mathrm{m}_{0}(\xi) & \mathrm{m}_{1}(\xi) \\
\mathrm{m}_{0}(\xi+1 / 2) & \mathrm{m}_{1}(\xi+1 / 2)
\end{array}\right]
$$

where $m_{0}$ and $m_{1}$ are trigonometric series called filters associated with $\varphi$ and $\psi$, respectively. The wavelet matrix $U$ is subject to constraints derived from the conditions and properties that $\varphi$ and $\psi$ must satisfy. Finding the coefficients of the filters $m_{0}$ and $m_{1}$ is essential to constructing a $(\varphi, \psi)$ pair. The following discussion revisits the wavelet feasibility problem which originally appeared in the work of Franklin, Hogan, and Tam [9, 10, 11].

MRA conditions and wavelet properties A consistency condition readily follows from (1), that is, $\mathrm{U}(\xi+1 / 2)=\sigma \mathrm{U}(\xi)$ where $\sigma$ is the 'row swap' matrix. Moreover, a necessary condition for the shifts and dilates of the wavelet to be orthonormal is that $\mathrm{m}_{0}(0)=1$ and $\mathrm{U}(\xi)$ is unitary almost everywhere. For $\varphi$ and $\psi$ to be compactly supported on $[0, M-1]$, we insist that $m_{0}$ and $m_{1}$ be trigonometric polynomials of the form $m_{0}(\xi)=\sum_{k=0}^{M-1} h_{k} e^{2 \pi i k \xi}$ and $m_{1}(\xi)=\sum_{k=0}^{M-1} g_{k} e^{2 \pi i k \xi}$. Consequently, $u(\xi)=\sum_{k=0}^{M-1} A_{k} e^{2 \pi i k \xi}$ where $A_{k} \in \mathbb{C}^{2 \times 2}$. Finally, the regularity requirement is achieved by requiring $\left.\frac{\mathrm{d}^{\ell}}{\mathrm{d} \xi^{\ell}} \mathrm{U}(\xi)\right|_{\xi=0}$ to be diagonal, for all $0<\ell \leqslant(M-2) / 2$. A more elaborate discussion about the foregoing design conditions is given by Franklin, Hogan, and Tam [11, Section 2.2].

Discretisation by uniform sampling The compact support condition allows for a discretisation of the problem by a uniform sampling at $M$ points $\{\mathrm{j} / \mathrm{M}\}_{j=0}^{\mathrm{M}-1} \subset[0,1)$. If $\mathrm{U}_{\mathrm{j}}=\mathrm{U}(\mathrm{j} / \mathrm{M})$, then the sampling procedure produces an ensemble of matrices $\mathcal{U}=\left(\mathrm{U}_{0}, \mathfrak{U}_{1}, \ldots, \mathrm{U}_{M-1}\right) \in\left(\mathbb{C}^{2 \times 2}\right)^{M}$. Note that the 
collection of coefficients $A_{k}$ may also be viewed as an ensemble $\mathcal{A}=\left(A_{k}\right)_{k=0}^{M-1}$. Moreover, we define an $M$-point discrete Fourier transform $\mathcal{F}_{M}:\left(\mathbb{C}^{2 \times 2}\right)^{M} \rightarrow$ $\left(\mathbb{C}^{2 \times 2}\right)^{M}$ by

$$
\left(\mathcal{F}_{M} \mathcal{B}\right)_{j}:=\sum_{k=0}^{M-1} B_{k} e^{2 \pi i j k / M} \quad \text { with inverse }\left(\mathcal{F}_{M}^{-1} \mathcal{D}\right)_{j}:=\frac{1}{M} \sum_{k=0}^{M-1} D_{k} e^{-2 \pi i j k / M},
$$

where $\mathcal{B}=\left(B_{k}\right)_{k=0}^{M-1}$ and $\mathcal{D}=\left(D_{k}\right)_{k=0}^{M-1} \in\left(\mathbb{C}^{2 \times 2}\right)^{M}$. With this transform, we have $\mathcal{U}=\mathcal{F}_{M} \mathcal{A}$ and $\mathcal{A}=\mathcal{F}_{M}^{-1} \mathcal{U}$. This establishes a connection between the uniform samples of $U(\xi)$ and the coefficient matrices $A_{k}$. A more comprehensive discussion on sampling and discretisation appeared in the work of Franklin, Hogan, and Tam [11, Section 3.2-3.3].

Wavelet properties encoded on the ensembles of matrices The consistency condition is imposed on the ensemble of samples to satisfy $\mathrm{U}_{\mathrm{j}+\frac{\mathrm{M}}{2}}=\sigma \mathrm{U}_{\mathrm{j}}$ for all $\mathrm{j} \in\{0,1, \ldots, M-1\}$. On the other hand, unitarity of each sample $U_{j}=U(j / M)$ for $j \in\{0,1, \ldots, M-1\}$ is insufficient to ensure the unitarity of $\mathrm{U}(\xi)$ almost everywhere. However, forcing $\mathrm{U}(\xi)$ to be unitary at $2 M$ samples, uniformly chosen to be $U(j / M)$ and $U((2 j+1) /(2 M))$, for $j \in\{0,1, \ldots, M-1\}$, is sufficient. Incidentally, given $\mathcal{U}=(\mathrm{U}(\mathrm{j} / \mathrm{M}))_{j=0}^{\mathrm{M}-1}$, the other ensemble $\tilde{\mathcal{U}}=[\mathrm{U}((2 j+1) /(2 \mathrm{M}))]_{j=0}^{\mathrm{M}-1}$ may be obtained entirely from the original samples in $\mathcal{U}$. Defining a modulation operator $\chi_{M}:\left(\mathbb{C}^{2 \times 2}\right)^{M} \rightarrow$ $\left(\mathbb{C}^{2 \times 2}\right)^{M}$ by $\left(\chi_{M} \mathcal{D}\right)_{k}=e^{\pi i k / M} D_{k}$ where $\mathcal{D}=\left(D_{k}\right)_{k=0}^{M-1} \in\left(\mathbb{C}^{2 \times 2}\right)^{M}$, we have

$$
\mathrm{U}\left(\frac{2 j+1}{2 M}\right)=\sum_{k=0}^{M-1} e^{2 \pi i j k / M} e^{\pi i k / M} A_{k}=\sum_{k=0}^{M-1} e^{2 \pi i j k / M}\left(\chi_{M} \mathcal{A}\right)_{k}=\left[\mathcal{F}_{M} \chi_{M} \mathcal{F}_{M}^{-1}(\mathcal{U})\right]_{j}
$$

Thus, $\tilde{\mathcal{U}}=\mathcal{F}_{M} \chi_{M} \mathcal{F}_{M}^{-1}(\mathcal{U})$. Lastly, the regularity condition is written in terms of the sample matrices $U_{k}$ as

$$
\left.\frac{d^{\ell}}{d \xi^{\ell}} \mathrm{U}(\xi)\right|_{\xi=0}=(2 \pi i)^{\ell} \sum_{j=0}^{M-1} j^{\ell} A_{j}=\sum_{k=0}^{M-1} \alpha_{\ell k} u_{k}
$$

where $\alpha_{\ell k}:=(2 \pi i)^{\ell} / M \sum_{j=0}^{M-1} j^{\ell} e^{-2 \pi i k j / M}$. 
Wavelet feasibility problem Let $\left(\mathbb{C}^{2 \times 2}\right)_{\sigma}^{M}$ denote the collection of ensembles in $\left(\mathbb{C}^{2 \times 2}\right)^{M}$ that satisfy the consistency condition. Furthermore, let $\mathbb{U}(2)$ denote the collection of all 2-by-2 unitary matrices, and $\mathbb{C} \otimes \mathbb{C}$ be the set of 2-by-2 diagonal matrices. The wavelet feasibility problem is stated as follows.

Definition 1. (Wavelet feasibility problem) Let $C_{1}, C_{2}, C_{3} \subseteq\left(\mathbb{C}^{2 \times 2}\right)_{\sigma}^{M}$ where $M$ is even and $M \geqslant 4$. The wavelet feasibility problem aims to find a $\mathcal{U}=\left(\mathrm{U}_{0}, \ldots, \mathrm{U}_{M-1}\right) \in \mathrm{C}_{1} \cap \mathrm{C}_{2} \cap \mathrm{C}_{3} \subseteq\left(\mathbb{C}^{2 \times 2}\right)_{\sigma}^{\mathrm{M}}$ where

$$
\begin{aligned}
& C_{1}:=\left\{\mathcal{U}: \mathrm{U}_{0}=\left(\begin{array}{ll}
1 & 0 \\
0 & z
\end{array}\right),|z|=1, \mathrm{u}_{j} \in \mathbb{U}(2), j \in\{0,1, \ldots, M / 2\}\right\}, \\
& \mathrm{C}_{2}:=\left\{\mathcal{U}:\left[\mathcal{F}_{M} \chi_{M} \mathcal{F}_{M}^{-1}(\mathcal{U})\right]_{j} \in \mathbb{U}(2), j \in\{0,1, \ldots, M / 2\}\right\}, \\
& C_{3}:=\left\{U: \sum_{k=0}^{M-1} \alpha_{\ell k} U_{k} \in \mathbb{C} \otimes \mathbb{C}, 1 \leqslant \ell \leqslant(M-2) / 2\right\} .
\end{aligned}
$$

We note that $C_{1}$ and $C_{2}$ are nonconvex subsets of $\left(\mathbb{C}^{2 \times 2}\right)_{\sigma}^{M}$, whereas $C_{3}$ is a subspace. A feasible point $\mathcal{U}=\left(\mathrm{U}_{0}, \ldots, \mathrm{U}_{M-1}\right)$ contains the $M$ samples of $U(\xi)$ from which we obtain the coefficients $A_{k}$ via $\mathcal{A}=\mathcal{F}_{M}^{-1} \mathcal{U}$. Consequently, the coefficients of the scaling filter $\mathrm{m}_{0}(\xi)$ and wavelet filter $\mathrm{m}_{1}(\xi)$ may be easily pulled out from the $A_{k}$. Through a cascade algorithm applied on the coefficients of $m_{0}(\xi)$ and $m_{1}(\xi)$, we may be able to plot the scaling function $\varphi$ and wavelet $\psi$, respectively.

\section{DR and CRM for wavelet construction}

In Franklin's work on wavelets $[9,10,11]$, DR solved more of the test cases than other projection algorithms, and so wavelets pose a natural setting for us to compare the performance of DR with that of CRM.

We first recall the operators associated with the DR and CRM algorithms. Let $\mathcal{H}$ be a real Hilbert space. For a closed subset $\mathrm{C}$ of $\mathcal{H}$, we define the 
operator $\mathrm{P}_{\mathrm{C}}: \mathcal{H} \rightarrow \mathrm{C}$ by $\mathrm{P}_{\mathrm{C}}(\mathrm{x})=\operatorname{argmin}_{z \in \mathrm{C}}\|z-x\|$; it is a selector for the closest point projection for $\mathrm{C}$. Its associated reflector is defined as $R_{V}:=2 P_{V}-I d$ where Id is the identity map. Given three points $x, y, z \in \mathcal{H}$, we denote $C(x, y, z)$ to be their circumcenter which is a point equidistant to $x, y, z$ and lies on the affine subspace that they define. ${ }^{1}$

Definition 2. Let $V$ and $W$ be subsets of $\mathcal{H}$ with nonempty intersection.

1. The DR operator is defined as $T(x):=x-P_{V}(x)+P_{W} R_{V}(x)$.

2. The circumcentering reflections method operator is defined as

$$
\operatorname{CRM}(x):=C\left(x, R_{V}(x), R_{W} R_{V}(x)\right) .
$$

3. The generically proper CRM operator is defined as

$$
C_{V, W}(x):= \begin{cases}T(x) & \text { if } x, R_{V} x, R_{W} R_{V} x \text { are colinear } \\ \operatorname{CRM}(x) & \text { otherwise. }\end{cases}
$$

Notice that the wavelet feasibility problem in Definition 1 has three constraint sets. Using a product space reformulation [17], it is rewritten as a two-set problem by defining the sets

$$
\begin{aligned}
S & :=C_{1} \times C_{2} \times C_{3}, \\
D & :=\left\{\left(\mathcal{U}_{1}, \mathcal{U}_{2}, \mathcal{U}_{3}\right): \mathcal{U}_{1}=\mathcal{U}_{2}=\mathcal{U}_{3} \in\left(\mathbb{C}^{2 \times 2}\right)_{\sigma}^{M}\right\} .
\end{aligned}
$$

The projectors onto $\mathrm{C}_{1}, \mathrm{C}_{2}$ and $\mathrm{C}_{3}$ are computed by Franklin [9, Chapter 6 ] while the projectors onto $S$ and $\mathrm{D}$ are given by Pierra [17, Section 1].

${ }^{1}$ When $\{x, y, z\}$ is a doubleton, the circumcenter is the average of the two distinct points, and when $\{x, y, z\}$ is a singleton, the circumcenter is the unique point. 
Table 1: Statistics of full run (not two-stage) DR and CRM performance as applied to the wavelet feasibility problem initialized at 1000 random points.

\begin{tabular}{lrr}
\multicolumn{1}{c}{ Algorithm } & DR & CRM \\
\hline cases solved & 986 & 316 \\
cases solved alone & 674 & 4 \\
wins when both converged & 38 & 274
\end{tabular}

Numerical Experiment: DR versus CRM In what follows, we attempt to solve the product space reformulation of the wavelet feasibility problem corresponding to the case where $M=6$. We confine our choice of projection algorithms to DR and CRM-where CRM here refers to its generically proper version. Henceforth, we let $\left\{x^{k}\right\}$ be the sequence of iterates generated by a projection algorithm. In all numerical implementations, we consider a tolerance $\epsilon:=10^{-9}$ and employ a stopping criterion given by $\| P_{D} P_{S}\left(x_{k}\right)-$ $\mathrm{P}_{\mathrm{S}}\left(\mathrm{x}_{\mathrm{k}}\right) \|<\epsilon$.

We consider a projection algorithm to have solved our feasibility problem if and when it attains a point that satisfies the stopping criterion within the cutoff of 100000 iterates. To present our numerical results, we plot errors given by $\left\|x^{k}-x^{k-1}\right\|$ and $\left\|P_{S}\left(x^{k}\right)-P_{S}\left(x^{k-1}\right)\right\|$ for the original and shadow sequences, respectively. We also provide statistics on the number of iterations which we mainly consider as a performance measure. Additionally, we comment on the versatility of an algorithm in tackling the nonconvex wavelet feasibilty problem by counting the number of times it solves a particular problem initialized at random starting points.

Table 1 summarises the performance of DR and CRM applied to 1000 random starting points. Initialised at similar starting points, DR demonstrates superior robustness to CRM. Interestingly, there are four starting points where DR failed to solve the problem but CRM succeeded. For all cases where DR and CRM both solved the problem, we compare the number of iterations needed and notice that CRM is more efficient than DR in most runs. Figure 1a shows a specific case where CRM failed but DR converged. The plot also highlights 


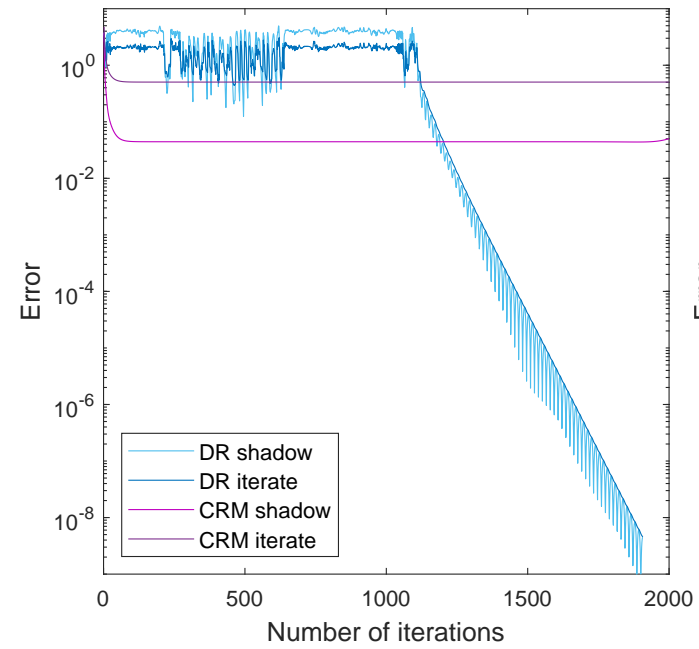

(a) DR versus CRM

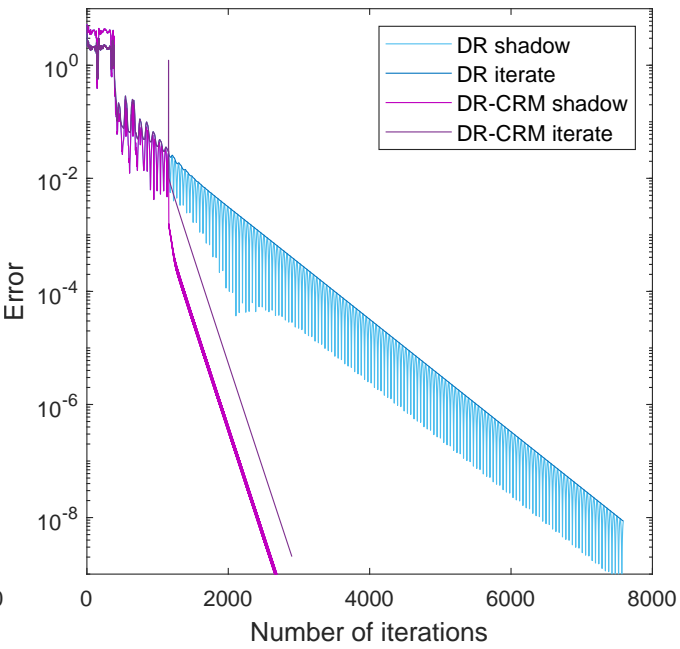

(b) DR versus DR-CRM

Figure 1: Performance comparisons of DR against CRM, and DR against two-stage DR-CRM in solving the wavelet feasibility problem with $M=6$ initialized at 1000 random points.

the propensity of DR to wander for a number of iterates before spiraling towards a feasible point. In the next section, we set up a two-stage DR-CRM to combine the apparent robustness of DR with the local speed of CRM.

Even when DR and CRM are initialized at the same starting point, the two approaches may yield different wavelets and scaling functions. In feasibility problems with more than one solution, the final destination of DR is known to exhibit high sensitivity to starting points [4, 12]. Since CRM behaves differently than DR, it is not surprising that the two methods may yield convergence to different feasible points from the same starting point. 


\section{The two-stage DR-CRM search algorithm}

Our experiments in Section 3 indicate that CRM may fail to locate a feasible point when it is used from the outset; that is, when a full run of plain CRM is used (see Figure 1a for an example). However, if both DR and CRM converge, CRM needed fewer iterations in most cases tested. This motivates us to assemble a two-stage DR-CRM to combine the apparent robustness of DR and the local speed of CRM. The two-stage DR-CRM is as follows.

1. Search for an attractive basin by iteratively applying $T_{S, D}(D R)$, and monitor the error $\left\|P_{D}\left(x_{n}\right)-P_{D}\left(x_{n-1}\right)\right\|$ for the oscillations that indicate spiralling.

2. Once oscillations are seen, switch to iteratively applying $\mathrm{C}_{S, \mathrm{D}}(\mathrm{CRM})$.

Numerical experiment: DR versus DR-CRM In implementing the twostage DR-CRM, we first run DR until the gap distance $\left\|P_{D} P_{S}\left(x^{k}\right)-P_{S}\left(x^{k}\right)\right\|$ reaches a $10^{-2}$ threshold, whereabouts we suspect DR to start spiralling in a local basin of attraction to a fixed point. Thereafter, we switch to running CRM until the stopping criterion is satisfied. For an example, we refer to Figure 1b. The threshold is reached after 552 iterations and it only took another 1745 iterations for CRM to satisfy the stopping criterion. We also plot a full run of DR in the same figure; DR took 7112 iterations beyond the 552 initial iterates to satisfy our stopping rule.

For the same set of random starting points used to obtain the data in Table 1, we generate another set of statistics to compare the performance of DR and the two-stage DR-CRM. To make a sensible analysis, we ignore the iterates needed to reach the $10^{-2}$ threshold and only present the statistics for both DR and the two-stage DR-CRM generated after threshold is obtained. Essentially, we are analyzing the action of DR and CRM, but only after a suitable basin is suspected to be reached. The statistics are summarized in Table 2. Notice that the two-stage DR-CRM outperformed DR in every test case, without introducing any loss of stability. The two-stage DR-CRM 
Table 2: Performance of DR and two-stage DR-CRM when applied to the wavelet feasibility problem initialized at 1000 random points.

\begin{tabular}{lrrrrrrrr} 
algorithm & solves & wins & min & Q1 & mean & Q3 & median & max \\
\hline DR & 986 & 0 & 560 & 697 & 5633 & 8162 & 6619 & 48683 \\
DR-CRM & 986 & 986 & 117 & 155 & 1093 & 1737 & 1612 & 1896
\end{tabular}

took at most 1896 iterations and a mean number of 1093 iterations. This is substantially smaller than the DR mean of 5633 iterations.

\section{Conclusion}

We have introduced a two-stage search for solving feasibility problems. The assembly of DR followed by CRM is motivated by the idea of combining the robustness of DR in searching for a feasible point and the ability of CRM to take bolder steps locally. Numerical experiments indicate that CRM may fail to locate the feasible point if it is used from the outset, prior to observed spiralling. However, CRM solved every problem when it was started after oscillations were observed in the error of DR method. The two-stage DR-CRM is seen to be faster in all cases than DR on its own. For this reason, we note that the two-stage method is the new state-of-the-art heuristic for solving wavelet feasibility problems on the line.

\section{References}

[1] F. J. Aragón Artacho, R. Campoy, and M. K. Tam. "The Douglas-Rachford algorithm for convex and nonconvex feasibility problems". In: Math. Meth. Oper. Res. 91 (2020), pp. 201-240. DOI: 10.1007/s00186-019-00691-9 (cit. on p. C100). 
[2] R. Behling, J. Y. Bello Cruz, and L.-R. Santos. "Circumcentering the Douglas-Rachford method". In: Numer. Algor. 78.3 (2018), pp. 759-776. DOI: 10.1007/s11075-017-0399-5 (cit. on p. C100).

[3] R. Behling, J. Y. Bello-Cruz, and L.-R. Santos. "On the linear convergence of the circumcentered-reflection method". In: Oper. Res. Lett. 46.2 (2018), pp. 159-162. ISSN: 0167-6377. DOI: 10.1016/j.orl.2017.11.018 (cit. on p. C100).

[4] J. M. Borwein, S. B. Lindstrom, B. Sims, A. Schneider, and M. P. Skerritt. "Dynamics of the Douglas-Rachford method for ellipses and p-spheres". In: Set-Val. Var. Anal. 26 (2018), pp. 385-403. DOI: 10.1007/s11228-017-0457-0 (cit. on pp. C100, C106).

[5] J. M. Borwein and B. Sims. "The Douglas-Rachford algorithm in the absence of convexity". In: Fixed-point algorithms for inverse problems in science and engineering. Springer, 2011, pp. 93-109. DOI: 10.1007/978-1-4419-9569-8_6 (cit. on p. C100).

[6] I. Daubechies. "Orthonormal bases of compactly supported wavelets". In: Commun. Pure Appl. Math. 41.7 (1988), pp. 909-996. DOI: 10.1002/cpa.3160410705 (cit. on p. C99).

[7] N. D. Dizon, J. A. Hogan, and J. D. Lakey. "Optimization in the construction of nearly cardinal and nearly symmetric wavelets". In: 13th International conference on Sampling Theory and Applications (SampTA). 2019, pp. 1-4. DOI: 10.1109/SampTA45681.2019.9030889 (cit. on p. C99).

[8] N. D. Dizon, J. A. Hogan, and S. B. Lindstrom. "Circumcentering reflection methods for nonconvex feasibility problems". In: arXiv preprint arXiv:1910.04384 (2019). URL:

https://arxiv.org/abs/1910.04384 (cit. on p. C100).

[9] D. J. Franklin. "Projection algorithms for non-separable wavelets and Clifford Fourier analysis". PhD thesis. University of Newcastle, 2018. DOI: 1959.13/1395028. (Cit. on pp. C99, C101, C103, C104). 
[10] D. J. Franklin, J. A. Hogan, and M. K. Tam. "A Douglas-Rachford construction of non-separable continuous compactly supported multidimensional wavelets". In: arXiv preprint arXiv:2006.03302 (2020). URL: https://arxiv.org/abs/2006.03302 (cit. on pp. C99, C101, C103).

[11] D. J. Franklin, J. A. Hogan, and M. K. Tam. "Higher-dimensional wavelets and the Douglas-Rachford algorithm". In: 13th International conference on Sampling Theory and Applications (SampTA). 2019, pp. 1-4. DOI: 10.1109/SampTA45681.2019.9030823 (cit. on pp. C99, C101, C102, C103).

[12] B. P. Lamichhane, S. B. Lindstrom, and B. Sims. "Application of projection algorithms to differential equations: Boundary value problems". In: ANZIAM J. 61.1 (2019), pp. 23-46. DOI: 10.1017/S1446181118000391 (cit. on pp. C100, C106).

[13] S. B. Lindstrom and B. Sims. "Survey: Sixty years of Douglas-Rachford". In: J. Aust. Math. Soc. 110 (2020), 1-38. DOI: 10.1017/S1446788719000570 (cit. on p. C100).

[14] S. B. Lindstrom, B. Sims, and M. P. Skerritt. "Computing intersections of implicitly specified plane curves". In: J. Nonlin. Convex Anal. 18.3 (2017), pp. 347-359. URL:

http://www. yokohamapublishers.jp/online2/jncav18-3 (cit. on p. C100).

[15] S. G. Mallat. "Multiresolution approximations and wavelet orthonormal bases of L ${ }^{2}(\mathbb{R})$ ". In: Trans. Amer. Math. Soc. 315.1 (1989), pp. 69-87. DOI: 10.1090/S0002-9947-1989-1008470-5 (cit. on p. C99).

[16] Y. Meyer. Wavelets and operators. Cambridge University Press, 1993. DOI: $10.1017 / \mathrm{CB} 09780511623820$ (cit. on p. C99).

[17] G. Pierra. "Decomposition through formalization in a product space". In: Math. Program. 28 (1984), pp. 96-115. DOI: 10.1007/BF02612715 (cit. on p. C104). 


\section{Author addresses}

1. N. D. Dizon, School of Information and Physical Sciences, University of Newcastle, New South Wales 2308, Australia.

mailto:neilkristofer.dizon@uon.edu.au

2. J. A. Hogan, School of Information and Physical Sciences, University of Newcastle, New South Wales 2308, Australia.

mailto: jeff.hogan@newcastle.edu.au

3. S. B. Lindstrom, Department of Applied Mathematics, The Hong Kong Polytechnic University, Kowloon, Hong Kong.

mailto: scott.lindstrom@curtin.edu.au 